\title{
Alcohol y diabetes: más evidencias sobre daños para la salud
}

\author{
César Pereiro Gómez \\ Unidad Asistencial de Drogodependencias de A Coruña. Plan de Galicia sobre Drogas. \\ Enviar correspondencia a: \\ César Pereiro Gómez. Unidad Asistencial de Drogodependencias de A Coruña (ACLAD). C) Ermita 2A, 15008 A Coruña. \\ e-mail: cesarpereiro@telefonica.net
}

$\mathbf{L}$ a diabetes mellitus (DM) es un trastorno metabólico crónico, caracterizado por niveles persistentemente elevados de glucosa en sangre, como consecuencia de una alteración en la secreción y/o acción de la insulina, que afecta además al metabolismo del resto de los hidratos de carbono, lípidos y proteínas. Se trata de un grave problema de salud pública derivado de su elevada frecuencia y de sus complicaciones crónicas, constituyendo una de las principales causas de invalidez y mortalidad, aparte de afectar a la calidad de vida de las personas afectadas. En Estados Unidos los pacientes con diabetes diagnosticada antes de los 15 años tienen una tasa de mortalidad 11 veces superior a la población general. La mortalidad es 2-3 veces mayor en pacientes en los que se diagnostica la enfermedad después de los 40 años. En la mayoría de los países desarrollados, la diabetes ocupa del $4^{\circ}$ al $8^{\circ}$ lugar entre las causas de defunción ${ }^{1}$. En España, según ha publicado el Grupo de Diabetes de la Sociedad Andaluza de Medicina Familiar y Comunitaria, representa la $3^{a}$ causa en mujeres y la $7^{a}$ en hombres $^{2}$

En el mundo occidental la prevalencia de diabetes mellitus se estima que oscila entre el 2 y el $6 \%$ de la población, según los distintos estudios (diferencias en métodos de diagnóstico empleados, rangos de edad, etc...). También se afirma que por cada persona con diabetes conocida existe una con diabetes sin diagnosticar. La prevalencia de la DM tipo1 se estima en $0,2 \%$ de la población $(5-10 \%$ de las personas con diabetes) y para la DM tipo 2 se considera que se aproximaría a un 6\% de la población (90-95\% de las personas con diabetes), aumentando de forma significativa en relación a la edad llegando a alcanzar cifras entre el 10-15 \% en la población mayor de 65 años.

Desde hace años es conocida la relación entre la diabetes y el consumo de alcohol y en la literatura médica también se han publicado los resultados de investigaciones sobre sobre los supuestos beneficios para la salud, en relación con la prevención de la dia- betes tipo 2, relacionado con el consumo de cantidades moderadas de alcohol ${ }^{3,6}$. Lo cierto es que aunque algunos estudios muy rigurosos aportaron evidencias en este sentido, en algunos casos los propios autores han revisado sus hallazgos y realizado afirmaciones en los que matizan los mismos, no siempre bien explicados. Es el caso de uno de los estudios más citados, realizado por Lapidus et al, publicado en Diabetes Care en septiembre del año $2005^{7}$. En el mencionado trabajo de investigación, en el que se incluían casi 1500 mujeres concluye que el aparente efecto protector del alcohol desaparece cuando se incluye en los cálculos el peso corporal de las mujeres, elemento clave del riesgo de diabetes. Los efectos positivos del alcohol también se veían debilitados cuando los investigadores tenían en cuenta los hábitos de ejercicio y el estatus socioeconómicos.

Lo cierto es que cualquier evidencia que atribuya propiedades beneficiosas al alcohol es aprovechada por las industrias productoras de bebidas alcohólicas a través de su difusión masiva y reiterada en los medios de comunicación y eso puede contribuir a la confusión de la población destinataria de los mensajes que no siempre analiza con la suficiente cautela y rigor el contenido de los mismos.

En relación con lo anteriormente mencionado, sobre el consumo de alcohol como factor que puede condicionar la mortalidad en los diabéticos, merece la pena destacar la publicación del artículo incluido en este número de la revista Adicciones, firmado por Yu. E. Razvodovsky ${ }^{8}$, sobre el efecto de agregación entre el consumo de alcohol y la tasa de mortalidad por diabetes. En el mencionado artículo se argumenta de forma contundente, con una metodología estadística rigurosa, como se ha incrementado más de tres puntos la mortalidad, tanto en hombres como en mujeres, entre 1981 y 2001 para la población de Bielorrusia. El mencionado crecimiento se atribuye al incremento en el consumo de bebidas de alta graduación (vodka). Un aspecto muy interesante del artículo 
antes mencionado es el relativo al análisis que realiza sobre los factores que pueden haber condicionado el incremento en la venta de bebidas alcohólicas para determinados períodos de tiempo señalando los cambios sociales, económicos y políticos radicales que se han vivido en las repúblicas de la Unión Soviética. El autor hipotetiza sobre la angustia social derivada de los cambios antes mencionados como un factor que podría haber intervenido en el incremento del consumo de bebidas alcohólicas y simultáneamente se indica la posible participación de la situación psicológica a través del estrés (algo ya demostrado anteriormente) como un factor condicionante del agravamiento de la diabetes. Se señala, de todos modos, la necesidad de realizar estudios posteriores, con mayor número de observaciones, para ampliar la información relativa a la relación entre los factores antes comentados como condicionantes de un incremento de la mortalidad.

\section{REFERENCIAS}

1. Howard AA; Arnsten JJ; Gourevitch M. Effect of Alcohol Consumption on Diabetes Mellitus. A Systematic Review. Ann Intern Med 2004; 140: 211-219.
2. Grupo de Trabajo sobre Diabetes de la SAMFyC. Disponible en: http://www.cica.es/ samfyc/guia1.htm. [consultado el 17/07/06].

3. Wei M; Gibbons LW; Mitchell TL; Kampert JB; Blair SN. Alcohol intake and incidence of type 2 diabetes in men. Diabetes Care 2000; 23: 18-22.

4. Kao WH; Puddey IB; Boland LL; Watson RL and Brancati FL.Alcohol Consumption and the Risk of Type 2 Diabetes Mellitus: Atherosclerosis Risk in Communities Study. Am J Epidemiol 2001; 154: 748-757.

5. Carlsson S; Hammar N; Grill V and Jaakko Kaprio. Alcohol consumption and the incidence of type 2 diabetes: a 20-year follow-up of the Finnish Twin Cohort Study. Diabetes Care 2003, 26: 2785-2786.

6. Koppes LL; Dekker JM; Hendriks HF; Bouter LM; Heine RJ: Moderate alcohol consumption lowers the risk of type 2 diabetes: a meta-analysis of prospective observational studies. Diabetes Care 2005; 28: 719725.

7. Lapidus L; Bengtsson C; Bergfors E, Björkelund C, Spak $F$ and Lauren Lissner $L$. Alcohol intake among women and its relationship to diabetes incidence and all-cause mortality. Diabetes Care 2005; 28: 2230-2235, 2005.

8. Razvodovsky YE. El efecto de la agregación entre el consumo de alcohol y la tasa de mortalidad por diabetes mellitus. Adicciones 2006; 18: 275-282 